\title{
(1)
}

Eduardo Alcalde-Gutiérrez*

Antonio Calvo-Bernardino**

Jesús Paúl-Gutiérrez ${ }^{* *}$

\section{EL MERCADO MUNDIAL DE DIVISAS A LA LUZ DE LA ENCUESTA DE 2019}

Desde el año 1986 el Banco Internacional de Pagos de Basilea viene coordinando, con la participación creciente de los bancos centrales de muchos países del mundo, una encuesta trianual sobre los mercados de divisas. A esta encuesta España se incorporó en 1989, en la segunda, y desde entonces ha venido participando activamente. Esta creciente participación de los mercados nacionales permite disponer de un detallado conocimiento sobre la evolución, el volumen y la composición de este mercado. Precisamente, el objetivo de este artículo es analizar los principales resultados obtenidos en la reciente encuesta realizada en abril de 2019 , comparando los mismos con los alcanzados en encuestas anteriores.

Palabras clave: mercado de divisas, intermediaros financieros, banco central, Banco Internacional de Pagos.

Clasificación JEL: F31, F36, G15.

\section{Introducción, características y limitaciones de la encuesta sobre el mercado de divisas}

Se han cumplido ya casi 35 años desde que el Banco Internacional de Pagos (BIS, por sus siglas en inglés), en colaboración con los bancos centrales nacionales participantes, realiza trianualmente, en el mes de abril, una encuesta sobre las transacciones en el mercado mundial de divisas.

\footnotetext{
* Facultad de Derecho, Universidad CEU San Pablo.

** Facultad de Ciencias Económicas y Empresariales, Universidad CEU San Pablo.

Versión de marzo de 2020.

DOI: https://doi.org/10.32796/bice.2020.3122.6982
}

En este amplio periodo de tiempo se han producido avances significativos para mejorar la cantidad y la calidad de la información disponible, y así aproximarse al volumen real de las transacciones que se realizan en el mercado mundial de divisas'.

En su evolución, la realización de comparaciones entre los diferentes años de la encuesta exige una cierta prudencia, en tanto en cuanto han sido muy intensos los cambios que se han producido, pudiendo ser, pues, en cada $\triangleright$

1 No obstante, el mercado de divisas es un mercado más opaco que muchos otros mercados financieros, dado que está organizado como un mercado over the counter (OTC). Como ponen de manifiesto Schrimpf $y$ Sushko (2019a), en años recientes, los cambios en la estructura del mercado, debidos a la internalización de las transacciones entre determinados colectivos, han reducido aún más su visibilidad para cualesquiera otros participantes en el mercado. 
momento las condiciones de partida diferentes. Entre ellos, algunos de los más relevantes serían: la continua incorporación de bancos centrales participantes (desde los cuatro iniciales - Canadá, Estados Unidos, Reino Unido y Japón-, pocos, pero muy representativos del conjunto del mercado, hasta los más de cincuenta que lo vienen haciendo desde el año 2004); la creación del euro, con la desaparición continuada de divisas que se han ido integrando en la moneda única, desde las once que lo hicieron en 1999 hasta las actuales diecinueve; los derivados de la revolución tecnológica permanente, con la aparición y desarrollo de divisas virtuales, nuevas plataformas de negociación, o de entidades no bancarias, que operan en el sector denominado de banca en la sombra; los periodos de crisis generalizada, con efectos sobre el PIB mundial, que han afectado a una buena parte de los países más desarrollados, como la bancaria y financiera, que se inicia en 2007 , y cuyos efectos se han dejado sentir hasta hace pocos años; los fenómenos de crisis más particulares, que afectan a zonas o países determinados, con las incertidumbres que se generan además para todas estas transacciones; los temores que pueden producirse por efectos contagio, ya no solo de carácter económico, sino también en otros ámbitos (pongamos por caso el pánico reciente por la expansión del coronavirus desde China al resto del mundo, especialmente a Europa, de lo que estamos todavía lejos de conocer sus consecuencias generales, pero que ha provocado una fuerte caída de las bolsas de muchos países durante los meses de febrero y marzo de 2020, y en particular sobre este mercado de divisas); o los cambios políticos, con las decisiones de ciertos dirigentes en relación a facilitar o no los movimientos de capitales, volviendo a la senda de los gravámenes; por citar algunos de los más importantes, como hemos indicado.
A pesar de todo ello, el mercado de divisas es uno de los mercados financieros más importantes a nivel mundial. Baste subrayar como representativo, a partir de algunos de los datos que manejamos más adelante en el trabajo, que el volumen anual de intercambios supera en más de siete veces el PIB mundial, y que su tasa media de crecimiento supera en los periodos trianuales el de ese PIB, incluidos los periodos de desaceleración económica o de crisis o de cambios en los mercados en sus cifras, como ha sucedido, por ejemplo, a finales del siglo pasado, con la creación del euro y la desaparición de monedas nacionales representativas de este mercado, o la crisis surgida a partir de 2007, en que el mercado también se ha resentido.

Específicamente dentro del mercado de divisas también se han producido a lo largo de los años cambios relevantes, desde las informaciones iniciales centradas en operaciones de intercambio tradicionales, como las de contado o de plazo, hasta el crecimiento y la información relativa a los mercados de derivados, que se empieza a ofrecer a partir de 1995.

Por otro lado, debemos recordar que los resultados de las transacciones se derivan de una encuesta, de una gran fiabilidad, eso sí, pues se encuentran implicados, como veremos, más del $90 \%$ del total de las entidades participantes en el mercado; por tanto, siempre los resultados hay que observarlos con la aproximación lógica de no incluir a la totalidad de los países y entidades potencialmente integrantes de los intercambios de divisas.

Además de lo anterior, como ya hemos indicado en publicaciones precedentes ${ }^{2}$, a la hora de analizar los datos de este mercado hay que tener en cuenta algunas cuestiones $\triangleright$

\footnotetext{
2 Véase, por ejemplo, Calvo y Paúl (2008b).
} 
adicionales $^{3}$ : en primer lugar, que los resultados de los intercambios se obtienen por agregación, con ciertas correcciones por doble contabilización, de forma que hay que proceder a un doble ajuste contable para evitarla; por una parte, un ajuste nacional, pues las entidades informantes de un mismo país ofrecen su propia información de los intercambios, de manera que esta aparece duplicada en la recogida por el banco central nacional; por otra, un ajuste global, para las ofrecidas por entidades informantes de dos países distintos, pues, en el agregado ofrecido al Banco Internacional de Pagos aparecerían notificadas dos veces, una por cada banco central informante; en segundo lugar, que las cifras se ofrecen en dólares estadounidenses, de forma que la comparación entre años, ya sean de operaciones concretas o de las cifras globales, están condicionadas por la evolución del tipo de cambio del dólar frente al resto de divisas, de manera que una depreciación de la moneda americana sobre las demás supondría que los intercambios totales en dólares fueran mayores, ocurriendo lo contrario en el caso de una apreciación de esa divisa de referencia, y, si fuera así, el resultado global sería consecuencia de la evolución del tipo de cambio del dólar y no del aumento o disminución real del mercado; tercero, que los cambios en el número de participantes y el nivel de cobertura de operaciones con la encuesta ha variado, lo que afecta sin duda alguna a las cifras globales del mercado.

Teniendo en cuenta los condicionantes anteriores, el presente artículo analiza, a grandes rasgos, la evolución, con especial hincapié en los últimos resultados de la encuesta trianual, del mercado de divisas mundial, haciendo referencia a la importancia en el conjunto de las

\footnotetext{
3 Recogemos aquí simplemente algunas de las más significativas.
}

diferentes divisas internacionales, a las intercambiadas en el mercado, los instrumentos utilizados, distinguiendo entre operaciones al contado ${ }^{4}$, plazo simple ${ }^{5}$ y swap de divisas ${ }^{6}$, que se correspondería con el mercado más tradicional, y opciones sobre divisas y currency swaps, que integrarían los derivados, así como a las contrapartidas institucionales de las operaciones, distinguiendo entre entidades básicamente de carácter bancario (o dealers informantes) ${ }^{7}$, otras instituciones financieras ${ }^{8}$ e instituciones no financieras ${ }^{9}$; a la importancia de los diferentes países (y de las diversas zonas geográficas) en el conjunto de este mercado; a la distribución en el tiempo de las operaciones a plazo; o el carácter geográfico de la operación, y no del mercado, distinguiendo entre operaciones locales $^{10}$ y de cross-border ${ }^{11}$.

4 Son operaciones al contado aquellas cuya liquidación de las mismas se efectúa, como máximo, dos días hábiles después de su contratación.

5 En las operaciones a plazo el periodo desde la contratación hasta la liquidación supera los dos días hábiles del mercado.

6 Las operaciones swaps incorporan una operación al contado y una a plazo simultáneamente de sentido contrario. Se trata, pues, de operaciones dobles, en las que se produce una compra o una venta de una divisa a plazo con su correspondiente venta o compra al contado (spot-forward). Dentro de las operaciones swaps también se incluyen otras combinaciones, como las de dos operaciones a plazo (forwardforward, realizadas en el mismo momento del tiempo, pero con vencimientos diferentes, superiores ambas a dos días hábiles del mercado), o las operaciones simultáneas, que se realizan ambas, como máximo, en dos días hábiles del mercado (tomorrow-next day).

7 Incluyen en la práctica todas las instituciones que participan en la encuesta, y que fundamentalmente son instituciones financieras muy activas en los mercados de divisas, como grandes bancos comerciales y de inversión, que participan como interdealers y/o tienen una actividad muy elevada con grandes clientes (Gobiernos, empresas u otras instituciones financieras).

8 Por exclusión se integran todas las no incluidas dentro de la categoría anterior; es decir, bancos comerciales o de inversión más pequeños, fondos de pensiones, compañías de seguros, entidades de leasing...

9 Se incluyen otros clientes no financieros, que no se integran en algunas de las dos categorías anteriores, como pueden ser las empresas no financieras o los Gobiernos.

10 Las operaciones locales se realizan entre dos agentes residentes, sin que tengan necesariamente la misma nacionalidad, y en cualquiera de las categorías de agentes que hemos indicado. Estas operaciones, por tanto, generan una doble contabilización, cuando son informadas por cada uno de los participantes, de carácter interno, y debe ser corregida por el banco central correspondiente.

11 En las operaciones cross-border uno de los participantes es un agente no residente en el país que ofrece la información. Estas operaciones generan una doble contabilización, cuando son informadas por cada uno de los participantes, de carácter externo, al informarse por dos bancos centrales distintos, debiéndose corregir por el Banco Internacional de Pagos, como agregador de toda la información. 
Eduardo Alcalde-Gutiérrez, Antonio Calvo-Bernardino y Jesús Paúl-Gutiérrez

CUADRO 1

PARTICIPANTES Y NIVEL DE REPRESENTATIVIDAD EN LA ENCUESTA SOBRE EL MERCADO MUNDIAL DE DIVISAS (2019)

\begin{tabular}{|c|c|c|c|}
\hline Países & Porcentaje de cobertura & Bancos que cubren el $75 \%$ & Número de participantes \\
\hline Alemania & 95 & 5 & 33 \\
\hline Arabia Saudí & 90 & 5 & 23 \\
\hline Argentina & 88 & 8 & 22 \\
\hline Australia & 96 & 8 & 23 \\
\hline Austria & 90 & 2 & 10 \\
\hline Bahrain & 100 & 6 & 30 \\
\hline Bélgica & 90 & 1 & 4 \\
\hline Brasil & 80 & 6 & 13 \\
\hline Bulgaria & 92 & 3 & 9 \\
\hline Canadá & 98 & 6 & 15 \\
\hline Chile & 98 & 6 & 27 \\
\hline China & 46 & 7 & 15 \\
\hline China Taipei & 88 & 17 & 30 \\
\hline Colombia & 95 & 11 & 17 \\
\hline Corea del Sur & 98 & 15 & 56 \\
\hline Dinamarca & 97 & 2 & 7 \\
\hline Emiratos Árabes Unidos & 88 & 7 & 11 \\
\hline Eslovaquia & 100 & 3 & 4 \\
\hline España & 96 & 3 & 4 \\
\hline Estados Unidos & 90 & 7 & 19 \\
\hline Filipinas & 100 & 13 & 30 \\
\hline Finlandia & 93 & 2 & 4 \\
\hline Francia & 99 & 4 & 44 \\
\hline Grecia & 93 & 3 & 4 \\
\hline Hong Kong SAR & 95 & 12 & 61 \\
\hline Hungría & 91 & 5 & 10 \\
\hline India & 85 & 12 & 23 \\
\hline Indonesia & 95 & 21 & 29 \\
\hline Irlanda & 100 & 4 & 14 \\
\hline Israel & 90 & 4 & 10 \\
\hline Italia & 96 & 4 & 16 \\
\hline Japón & 90 & 11 & 36 \\
\hline Letonia & 52 & 3 & 6 \\
\hline Lituania & 54 & 2 & 3 \\
\hline Luxemburgo & 100 & 12 & 132 \\
\hline Malasia & 80 & 9 & 10 \\
\hline México & 93 & 7 & 20 \\
\hline Noruega & 80 & 3 & 5 \\
\hline Nueva Zelanda & 99 & 2 & 4 \\
\hline Países Bajos & 95 & 2 & 5 \\
\hline Perú & 98 & 5 & 18 \\
\hline Polonia & 95 & 8 & 13 \\
\hline Portugal & 100 & 4 & 50 \\
\hline Reino Unido & 97 & 9 & 55 \\
\hline República Checa & 85 & 3 & 9 \\
\hline Rumanía & 99 & 5 & 28 \\
\hline Rusia & 99 & 9 & 55 \\
\hline Singapur & 99 & 12 & 80 \\
\hline Sudáfrica & 97 & 4 & 11 \\
\hline Suecia & 90 & 3 & 4 \\
\hline Suiza & 100 & 4 & 31 \\
\hline Tailandia & 100 & 12 & 28 \\
\hline Turquía & 96 & 6 & 16 \\
\hline Media y total & 91,51 & 347 & 1.236 \\
\hline
\end{tabular}


En el apartado final destacaremos algunas de las conclusiones más relevantes obtenidas a lo largo del trabajo.

\section{Evolución de los resultados de la encuesta global}

\subsection{Volumen del mercado}

En los primeros años de realización de la encuesta sobre las transacciones en el mercado de divisas se fue produciendo un aumento paulatino de su representatividad, estabilizándose la misma a partir de 2001, con una participación desde entonces de alrededor de 50 bancos centrales de todo el mundo, que supone, como podemos ver en el Cuadro 1 para la última realizada, un porcentaje de cobertura que supera el 91,5 del mercado, con casi 1.240 bancos participantes, de los que aproximadamente 350 de ellos superan una tasa de cobertura del $75 \%$ del total del mercado de su país.

Además, en los países con volúmenes de negociación más significativos, como son los casos de Reino Unido, Estados Unidos o Japón, las entidades participantes suponen una cuota del mercado que se sitúa en o por encima del $90 \%$ del total de su mercado. Solamente el mercado chino, entre los más relevantes, tiene todavía un nivel de participación de sus entidades reducido, menor al $50 \%$, solo aproximado a la que tiene el letón o el lituano, que supera ligeramente esa participación.

A pesar de todo ello, observamos un mercado con un crecimiento importante en media a lo largo de todo el periodo, como se comprueba en el Gráfico 1. De su contenido podemos destacar que la media de cifras diarias de transacciones en abril de 2019 se situó en 6,59 billones de dólares, la cantidad mayor desde que se realiza la encuesta, superando la disminución que se produjo en 2016, fruto de la $\triangleright$

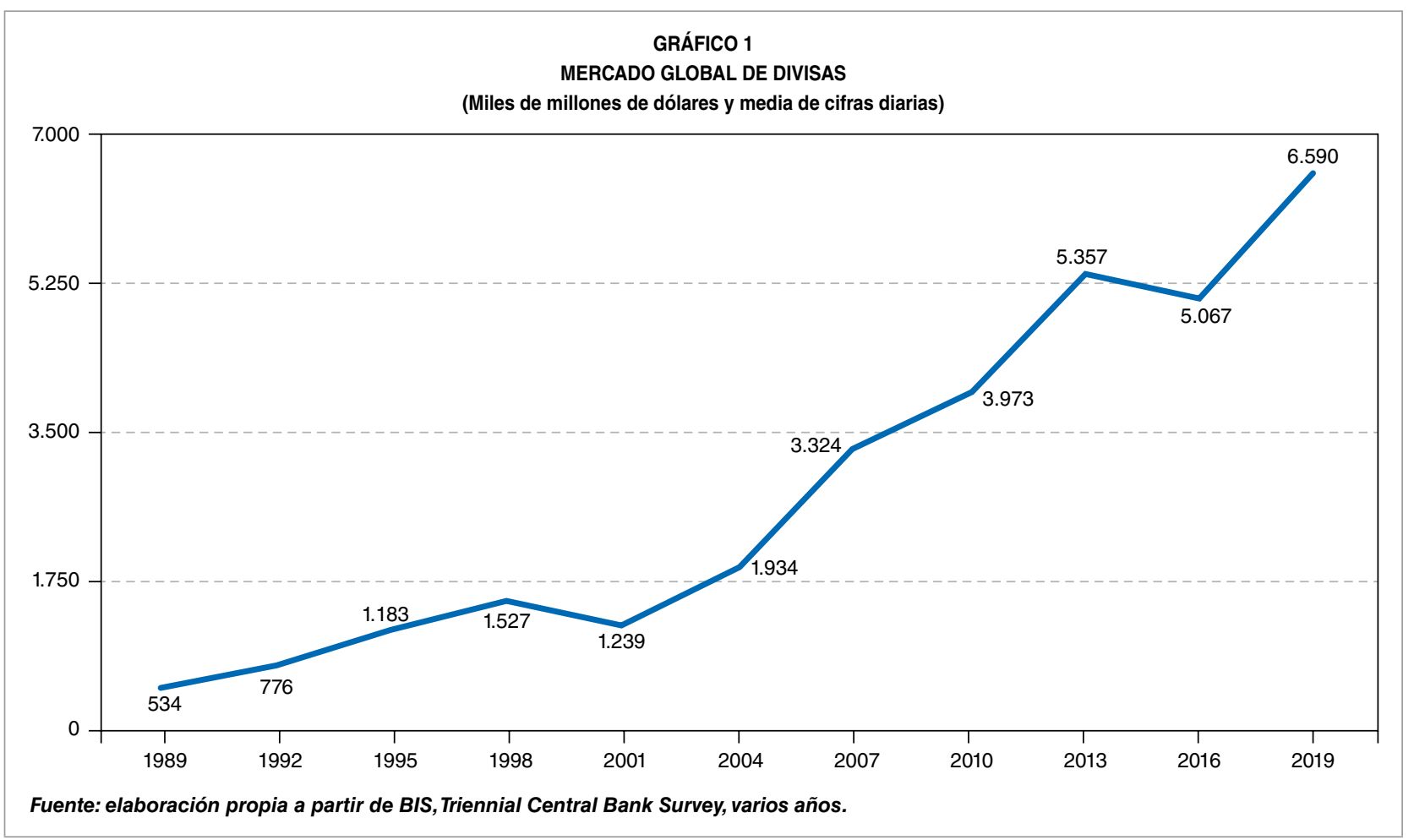


crisis económica y financiera reciente, y que ha permitido un crecimiento en estos tres años de 1,523 billones de dólares, a una tasa del $30 \%$, próxima a lo que ha sido el crecimiento medio anual de este mercado desde principios del siglo $x \mathrm{xI}$.

Sin embargo, este crecimiento no ha sido constante a lo largo de todo el periodo, sino que ha habido dos momentos en el tiempo, las encuestas correspondientes a los años 2001 y 2016, en las que se han producido caídas en el volumen global negociado, derivadas, en el primero de los periodos, por la desaparición de las monedas europeas integradas en el euro, por lo que se eliminaron las negociaciones que se producían entre ellas, por los procesos de concentración de entidades bancarias, que redujo el número de participantes en la encuesta, por los incrementos en la intermediación electrónica que restó importancia a la labor bancaria tradicional y por la globalización de los clientes no financieros, con presencia en una multitud de países, que convirtió en innecesarias algunas de las transacciones que antes se efectuaban. Por su parte, en 2016, además de esta continua globalización de los clientes no financieros, de la vertiginosa tecnificación o de la presencia de entidades financieras en cada vez más países del mundo, la crisis financiera de la pasada década ha hecho que el mercado se contrajera en algo más de un $5 \%$.

\subsection{Composición del mercado}

\subsubsection{Evolución de la importancia de cada divisa en el mercado}

Desde el inicio de la realización de la encuesta, el dólar estadounidense ha ocupado, con gran diferencia, el primer lugar dentro de las divisas intercambiadas, pues, como podemos comprobar en el Cuadro $2^{12}$, esta moneda aparece en más del $88 \%$ de los intercambios producidos, de forma muy estable además en todo lo que llevamos del siglo xxı (con una ligera caída del $1,78 \%$ ). Solamente el euro, con un tercio de los intercambios, se le aproxima, si bien desde 2010 ha perdido una cierta participación, pues en ese año entró en más de un $39 \%$ de los intercambios ${ }^{13}$. La crisis económica y financiera que ha afectado a Europa de forma diferencial en esta década pasada se encuentra en las razones de esta pérdida de relevancia del euro en las transacciones de este mercado. Solo el yen y la libra esterlina superan una participación del $10 \%$ en las transacciones de divisas de forma sistemática en todo el siglo XXI, si bien la moneda japonesa ha conocido una pérdida de cuota significativa en la última encuesta, al pasar de aparecer en más del $21 \%$ de las operaciones a solo hacerlo en el $16,8 \%$, alcanzando su participación máxima al inicio y en 2013 , con el $23 \%{ }^{14}$. En el caso de la libra, ha mantenido una cierta estabilidad a lo largo de esta última década, cayendo no obstante respecto a los resultados que alcanzó en la encuesta de 2004, cuando logró aparecer en el $16,5 \%$ de los intercambios ${ }^{15}$.

Respecto a las demás divisas, solo el dólar australiano $^{16}$, el canadiense y el franco suizo $\square$

12 Obsérvese que la suma total es 200 porque en toda transacción en este mercado aparecen dos divisas, por lo que las cifras están duplicadas.

13 De hecho, la caída en la presencia del euro en lo que llevamos del siglo XXI ha sido del $14,8 \%$, a pesar del aumento en la última encuesta respecto a la anterior $(2,9 \%)$.

14 La evolución de la moneda japonesa ha sido muy negativa, tanto en la última encuesta como en lo que se lleva de este siglo, pues en la última esa presencia se ha reducido en un $22,3 \%$, para una disminución en el conjunto del siglo $x x \mathrm{x}$, del $28,5 \%$.

15 Esta estabilidad en la presencia en los mercados de divisas de la libra se pone de manifiesto en que en este siglo su participación se ha reducido solo en $1,54 \%$, si bien con diferencias entre encuestas.

16 Se trata de la divisa que más ha crecido entre las más intercambiadas, pues lo ha hecho en más de un $58 \%$ en lo que va de siglo, solo superado ese crecimiento por varias de las monedas asiáticas (won coreano, rupia india, remminbi chino, nuevo dólar de Taiwán) o por el dólar neozelandés, si bien la participación de todas ellas todavía se sitúa a niveles bastante más reducidos que las de la moneda del país oceánico. 
CUADRO 2

DISTRIBUCIÓN DE LAS TRANSACCIONES POR MONEDAS EN EL MERCADO GLOBAL DE DIVISAS

(En porcentajes)

\begin{tabular}{|c|c|c|c|c|c|c|c|}
\hline Monedas & 2001 & 2004 & 2007 & 2010 & 2013 & 2016 & 2019 \\
\hline Dólar USA & 89,9 & 88,0 & 85,6 & 84,9 & 87,0 & 87,6 & 88,3 \\
\hline Euro & 37,9 & 37,4 & 37,0 & 39,1 & 33,4 & 31,4 & 32,3 \\
\hline Yen & 23,5 & 20,8 & 17,2 & 19,0 & 23,0 & 21,6 & 16,8 \\
\hline Libra esterlina & 13,0 & 16,5 & 14,9 & 12,9 & 11,8 & 12,8 & 12,8 \\
\hline Dólar australiano & 4,3 & 6,0 & 6,6 & 7,6 & 8,6 & 6,9 & 6,8 \\
\hline Dólar canadiense & 4,5 & 4,2 & 4,3 & 5,3 & 4,6 & 5,1 & 5,0 \\
\hline Franco suizo & 6,0 & 6,0 & 6,8 & 6,3 & 5,2 & 4,8 & 5,0 \\
\hline Renminbi & 0,0 & 0,1 & 0,5 & 0,9 & 2,2 & 4,0 & 4,3 \\
\hline Dólar de Hong Kong & 2,2 & 1,8 & 2,7 & 2,4 & 1,4 & 1,7 & 3,5 \\
\hline Dólar neozelandés & 0,6 & 1,1 & 1,9 & 1,6 & 2,0 & 2,1 & 2,1 \\
\hline Corona sueca & 2,5 & 2,2 & 2,7 & 2,2 & 1,8 & 2,2 & 2,0 \\
\hline Won coreano & 0,8 & 1,1 & 1,2 & 1,5 & 1,2 & 1,7 & 2,0 \\
\hline Dólar de Singapur & 1,1 & 0,9 & 1,2 & 1,4 & 1,4 & 1,8 & 1,8 \\
\hline Corona noruega & 1,5 & 1,4 & 2,1 & 1,3 & 1,4 & 1,7 & 1,8 \\
\hline Peso mexicano & 0,8 & 1,1 & 1,3 & 1,3 & 2,5 & 1,9 & 1,7 \\
\hline Rupia india & 0,2 & 0,3 & 0,7 & 1,0 & 1,0 & 1,1 & 1,7 \\
\hline Lira turca & 0,0 & 0,1 & 0,2 & 0,7 & 1,3 & 1,4 & 1,1 \\
\hline Rublo & 0,3 & 0,6 & 0,7 & 0,9 & 1,6 & 1,1 & 1,1 \\
\hline Real brasileño & 0,5 & 0,3 & 0,4 & 0,7 & 1,1 & 1,0 & 1,1 \\
\hline Rand sudafricano & 0,9 & 0,7 & 0,9 & 0,7 & 1,1 & 1,0 & 1,1 \\
\hline Nuevo dólar de Taiwán & 0,3 & 0,4 & 0,4 & 0,5 & 0,5 & 0,6 & 0,9 \\
\hline Corona danesa & 1,2 & 0,9 & 0,8 & 0,6 & 0,8 & 0,8 & 0,6 \\
\hline Zloty polaco & 0,5 & 0,4 & 0,8 & 0,8 & 0,7 & 0,7 & 0,6 \\
\hline Otras monedas & 7,5 & 7,7 & 9,1 & 6,4 & 4,4 & 4,9 & 5,6 \\
\hline Todas las monedas & 200,0 & 200,0 & 200,0 & 200,0 & 200,0 & 200,0 & 200,0 \\
\hline
\end{tabular}

tienen una participación en los intercambios que se sitúa por encima o al nivel del $5 \%$, aunque todas ellas alcanzaron sus mayores participaciones en encuestas anteriores. De todas las demás, es destacable el crecimiento continuado de las monedas asiáticas, especialmente el remminbi chino, que se sitúa en un $4,3 \%$ de las transacciones en la última encuesta, el dólar de Hong Kong, con el 3,5\% (duplicando su cuota en la de 2019), el won coreano o el dólar de Singapur, lo que demuestra un cierto giro del mercado mundial hacia la zona asiática ${ }^{17}$.

17 Schrimpf y Sushko (2019b) señalan que la concentración geográfica se debe en parte a la presencia de economías de red, por el menor coste que supone centralizar las contrapartidas y las relaciones crediticias, o las infraestructuras técnicas y legales, que distribuirlas entre países.
Por otra parte, cuando nos referimos a los pares de monedas intercambiadas, es lógico observar, a partir de los resultados recogidos en el cuadro anterior, que es el dólar de Estados Unidos con el euro el par de divisas que más aparece en las operaciones, como podemos comprobar en el Cuadro 3.

En dicho cuadro recogemos los principales intercambios en los que se encuentran involucrados el dólar norteamericano y el euro, como monedas que, a nivel individual, más aparecen en el mercado. Entre ellas, aunque con una reducción de 6 puntos porcentuales en sus transacciones conjuntas respecto a principios de siglo, sus operaciones representan aproximadamente la cuarta parte del total $D$ 
CUADRO 3

DISTRIBUCIÓN DE LAS TRANSACCIONES POR PARES DE MONEDAS EN EL MERCADO GLOBAL DE DIVISAS

(En porcentajes)

\begin{tabular}{|l|r|r|r|r|}
\hline & $\mathbf{2 0 0 1}$ & $\mathbf{2 0 1 3}$ & $\mathbf{2 0 1 6}$ & $\mathbf{2 0 1 9}$ \\
\hline Dólar USA/Euro & 30,0 & 24,1 & 23,1 & 24,0 \\
Dólar USA/Yen & 20,2 & 18,3 & 17,8 & 13,2 \\
Dólar USA/Libra esterlina & 10,4 & 8,8 & 9,3 & 9,6 \\
Dólar USA/Dólar austral & 4,1 & 6,8 & 5,2 & 5,4 \\
Dólar USA/Otras monedas & 20,8 & 29,0 & 32,2 & 36,2 \\
Dólar USA/monedas de economías de mercados emergentes & - & - & 17,1 & 20,2 \\
Euro/Yen & 2,9 & 2,8 & 1,6 & 1,7 \\
Euro/Libra esterlina & 2,1 & 1,9 & 2,0 & 2,0 \\
Euro/Otras monedas & 5,2 & 4,6 & 4,7 & 4,6 \\
\hline Fuente: elaboración propia a partir del BIS, Triennial Central Bank Survey, varios años. & & &
\end{tabular}

del mercado. También han caído de forma importante las que se realizan entre la divisa americana y el yen, resultado de la menor presencia de la divisa japonesa en las últimas encuestas y la aparición de las monedas de los países emergentes, que han entrado con fuerza en los intercambios, hasta suponer en la de 2019 una de cada cinco operaciones de las que realiza el dólar estadounidense ${ }^{18}$. La caída en la presencia del yen también es significativa en sus intercambios con el euro, cuya combinación ha perdido 1,2 puntos porcentuales en el mercado desde 2001.

\subsubsection{Operaciones y contrapartidas del mercado}

El importante incremento de las transacciones realizadas en el mercado de divisas, de acuerdo con la última encuesta, se ha producido en todos los instrumentos utilizados en el mercado. Tanto el mercado tradicional, compuesto por las operaciones al contado, a plazo simple y los swaps de divisas, como el global,

\footnotetext{
18 Mientras que las transacciones mundiales de divisas crecieron en la última encuesta un $33 \%$, los intercambios en los que estuvieron involucradas las monedas de países emergentes lo hicieron en casi un $60 \%$. Para un análisis detallado de las causas del crecimiento de estas monedas, puede verse Patel y Xia (2019).
}

al que se añaden las opciones sobre divisas y los currency swaps, experimentaron un aumento medio entre el 30-31\% respecto a 2016. Este aumento en todas las operaciones que lo integran fue más significativo, como podemos observar en el Cuadro 4, en las realizadas a plazo simple, con un $42,7 \%$ respecto a la encuesta anterior, y en los swaps de divisas, que lo hicieron en un $34,7 \%$. Incluso si tenemos en cuenta el año 2013, las operaciones a plazo, junto con los currency swaps, con cifras modestas, son las únicas modalidades que crecieron en los tres periodos, a pesar de la reducción en el global que se produjo en la encuesta de 2016.

En el Gráfico 2, por su parte, recogemos la evolución de la distribución de esos instrumentos en lo que llevamos del siglo xxı. De su contenido podemos destacar: en primer lugar, que las posiciones ocupadas por cada uno de ellos no han cambiado en todo el periodo, correspondiendo el primer puesto a las operaciones combinadas o swaps, que han supuesto en general, salvo en la última encuesta, alrededor del $50 \%$ de las operaciones efectuadas. La segunda posición la han ocupado las operaciones al contado, que se han movido entre el $30 \%$ y el $40 \%$ del mercado a lo largo del $\square$ 
CUADRO 4

TRANSACCIONES EN EL MERCADO MUNDIAL DE DIVISAS POR INSTRUMENTOY CONTRAPARTIDAS INSTITUCIONALES (Miles de millones de dólares y medias de cifras diarias)

\begin{tabular}{|c|c|c|c|c|c|}
\hline & 2012 & 2016 & 2010 & Variación d & últimos años \\
\hline & 2010 & 2010 & 2010 & Absoluta & Porcentual \\
\hline A. Por instrumento & & & & & \\
\hline 1. Contado & 2.047 & 1.652 & 1.987 & 335 & 20,3 \\
\hline 2. Plazo simple & 679 & 700 & 999 & 299 & 42,7 \\
\hline 3. Swaps de divisas & 2.240 & 2.378 & 3.202 & 824 & 34,7 \\
\hline Mercado Tradicional de Divisas $(1+2+3)$ & 4.966 & 4.730 & 6.188 & 1.458 & 30,8 \\
\hline 4. Opciones sobre divisas & 337 & 254 & 294 & 40 & 15,7 \\
\hline 5. Currency swaps & 54 & 82 & 108 & 26 & 31,7 \\
\hline Mercado Global de Divisas $(1+2+3+4+5)$ & 5.357 & 5.066 & 6.590 & 1.524 & 30,1 \\
\hline B. Por contrapartidas institucionales & & & & & \\
\hline 6. Dealers informantes & 2.072 & 2.121 & 2.522 & 401 & 18,9 \\
\hline 7. Otras instituciones financieras & 2.812 & 2.564 & 3.595 & 1.031 & 40,2 \\
\hline 8. Instituciones no financieras & 472 & 382 & 474 & 92 & 24,1 \\
\hline
\end{tabular}

periodo contemplado, lográndose la mayor participación, con el $37 \%$, en la encuesta de 2010. Tanto las operaciones a plazo como las de derivados en general tienen una importancia menor, aunque las primeras lograron un porcentaje en el total de operaciones del $15 \%$ en la última encuesta, habiéndose situado con anterioridad entre el $10 \%$ y el porcentaje anterior.

En segundo término, todas las operaciones, salvo las de plazo, han estado afectadas en su evolución por la tendencia general seguida por el mercado global. En efecto, mientras que estas últimas operaciones han ido ganando $D$

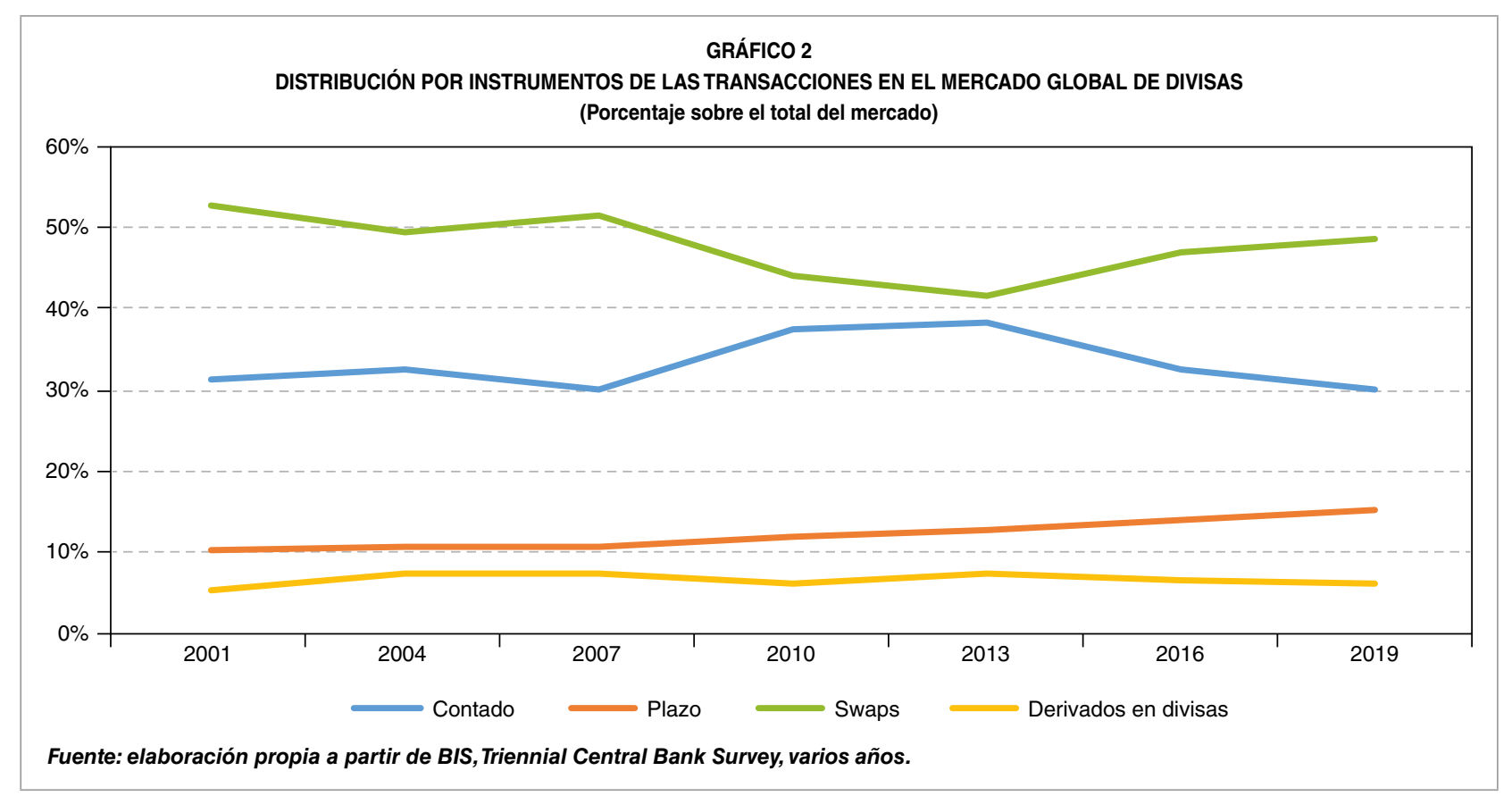


cuota de participación de forma continuada a lo largo de todas las encuestas realizadas en el presente siglo, en el resto la evolución ha sido discontinua, alcanzando sus puntos máximos de participación en años distintos al último; así, por ejemplo, los swaps lo alcanzan en la encuesta de 2001, las de contado en 2013, cayendo a partir de entonces de forma significativa, además. Finalmente, el Gráfico 2 resulta muy ilustrativo al poderse comprobar que las operaciones al contado y swap se han movido de forma distinta en todo el periodo, de manera que cuando la participación de una de ellas crecía, lo hacía generalmente a costa de la otra, lo cual constata en general la estabilidad de las cifras de las demás operaciones.

Dado pues que las operaciones a plazo, combinadas fundamentalmente, son las que alcanzan las mayores cifras, con el fin de profundizar en su distribución temporal, recogemos en el Cuadro 5 la misma, diferenciando, en primer lugar, entre las realizadas a plazo simple de las de swaps de divisas, y en segundo, por vencimiento de las operaciones, ya sea de hasta una semana o por más de ese plazo, para las tres últimas encuestas efectuadas.
Resulta significativo que, por lo general, en el mercado de divisas, dentro del predominio ya destacado de las operaciones swaps (76,2\% en el segmento a plazos), haya diferencias en los plazos según el tipo de operación realizada, pues mientras que en las swaps predominan las operaciones de una semana o menos, es decir, que los intercambios, ya sean contado a plazo o ya sean plazo a plazo, el último vencimiento, y, por tanto, el cierre de la operación, es fundamentalmente de hasta siete días $(64,4 \%$ en la última encuesta); en las de plazo, que suponen una única operación, se realizan en un $73 \%$ de los casos a periodos superiores a la semana ${ }^{19}$.

$\mathrm{Si}$, como hicimos antes, tomamos como referencia todas las encuestas realizadas en el presente siglo, entonces sus resultados aparecen en el Gráfico 3.

De su contenido son varias las conclusiones que podemos alcanzar: en primer lugar, el predominio en todas las encuestas, con gran diferencia además de las operaciones swaps a $\triangleright$

19 Este aumento de las operaciones swap se facilitó por la abundante liquidez en la zona euro, que llevó a las entidades bancarias a la realización de operaciones combinadas de esta moneda fundamentalmente con el dólar de Estados Unidos y también en el intento de explotar los diferenciales de precios entre ellas (Schrimpf y Sushko, 2019a: 26-27).

CUADRO 5

MERCADO DE DIVISAS A PLAZO

(Miles de millones de dólares y media de cifras diarias en abril)

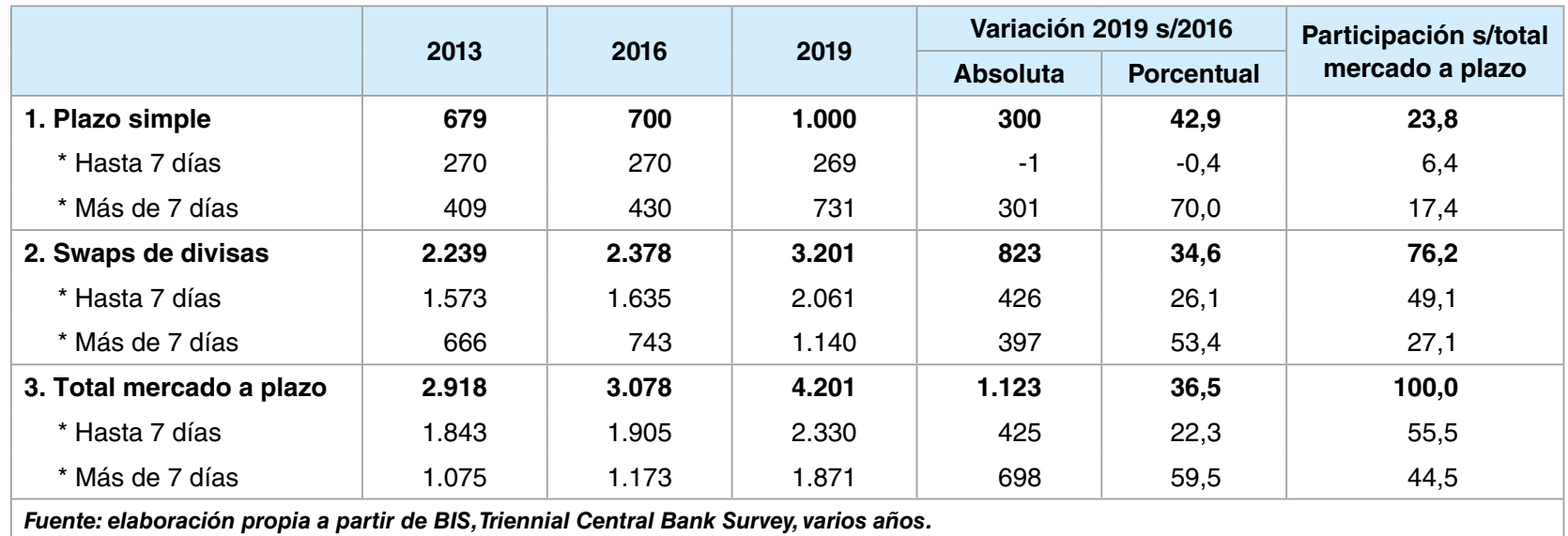




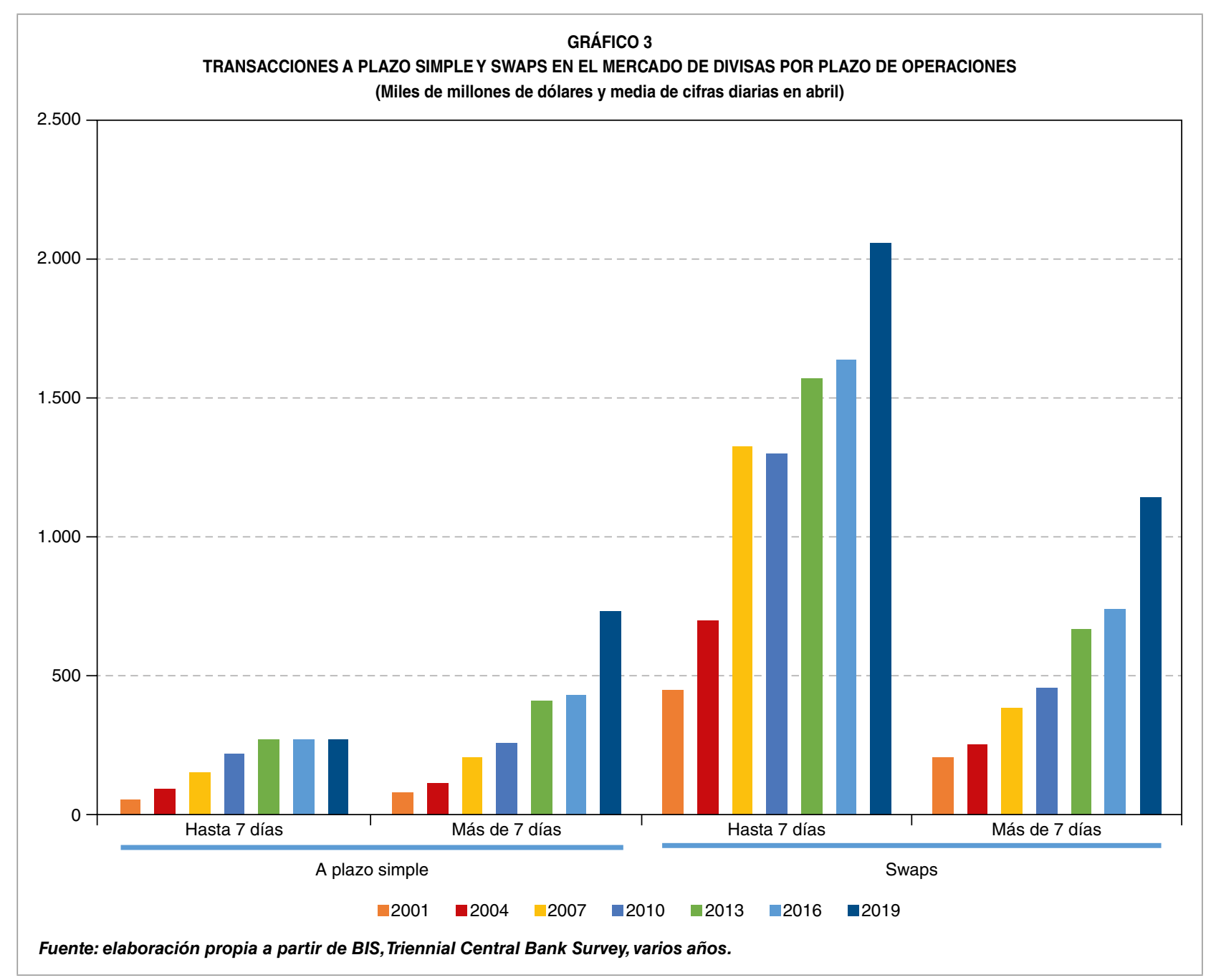

plazo de una semana o inferior; en segundo lugar, que incluso las de un plazo superior alcanzan en todo el periodo una cifra mayor a la de cualquiera de las modalidades de las de plazo simple; en tercer lugar, que, a pesar de que en el presente siglo ha habido dos periodos de descenso en las cifras del mercado global, las operaciones a plazo superior a una semana han crecido de forma continuada, de forma más significativa los swaps ${ }^{20}$.

20 Mientras que las operaciones a plazos inferiores han conocido un cierto descenso (poco relevante, eso sí, en alguna de las encuestas), concretamente las que alcanzan las cifras más elevadas, los swaps a una semana descendieron en 2010 , mientras que las de plazo a una semana lo hicieron mínimamente en la última encuesta, siendo muy estables sus cifras en las tres últimas.
En el Cuadro 4 anterior también recogíamos la información correspondiente a las contrapartidas institucionales de las operaciones realizadas en el mercado para las tres últimas encuestas. Solo los dealers informantes, las entidades de mayor tamaño que operan en los mercados de divisas, han mantenido su crecimiento en el conjunto de los tres periodos, hasta suponer el $38,3 \%$ del total de las operaciones intermediadas en el mercado. Más significativo aún resulta la evolución en esos tres años del resto de instituciones financieras, que siguieron la caída del mercado en 2016, pero que se han recuperado de forma significativa en 2019 , con un aumento en sus cifras $D$ 
intermediadas del 40,2\%, situándose, eso sí, en los tres periodos contemplados, en el primer lugar de las contrapartidas del mercado. Por su parte, las instituciones no financieras, aunque crecieron un $24,1 \%$ en la última encuesta respecto a la anterior, su participación en el conjunto global se mantiene en números muy pequeños, lo cual se viene constatando a lo largo de todo el presente siglo, como podemos ver también en el Gráfico $4^{21}$.

En efecto, esas instituciones no financieras, pese al repunte que se observa en 2019 , han ido perdiendo importancia relativa de forma continuada en el conjunto del mercado desde 2007, año que supusieron el $18 \%$ de las operaciones intermediadas; desde entonces su caída ha sido continua hasta situarse

21 Empiezan a aparecer, y con un crecimiento importante en el futuro, las fintech y las bigtech, que, con presencia global en el comercio y otras actividades, pueden condicionar el futuro del mercado. También se desarrollarán nuevas criptomonedas, amparadas o no por algunos bancos centrales más importantes, con efectos ahora que no es posible determinar sobre el conjunto del mercado. Véase, como un análisis reciente en los cambios de la actividad bancaria, Casilda (2020). en la última encuesta en el $7 \%$. Esta caída se ha producido fundamentalmente a favor de las otras instituciones financieras, pues desde el año en que lograron aquellas el máximo han ganado 15 puntos porcentuales, de los que, por tanto, en 2019, 4 puntos corresponden a la caída global en ese intervalo de los dealers informantes. Lo más relevante, sin duda, ha sido la continua pérdida de importancia de estos en el conjunto de la intermediación en el mercado, pues hasta la encuesta de 2007 fueron los que acapararon una mayor parte del mismo, llegando hasta el $58 \%$ en 2001 , perdiendo pues 20 puntos porcentuales en lo que va de siglo, que, junto a los 7 puntos perdidos por las instituciones no financieras, suponen los 27 de ganancia que ha tenido el resto de instituciones, que han duplicado la importancia relativa en los intercambios en el mercado en lo que llevamos del siglo xxı. Todo ello responde a la generalización de las plataformas electrónicas de negociación (Bank of International Settlements, $\triangleright$

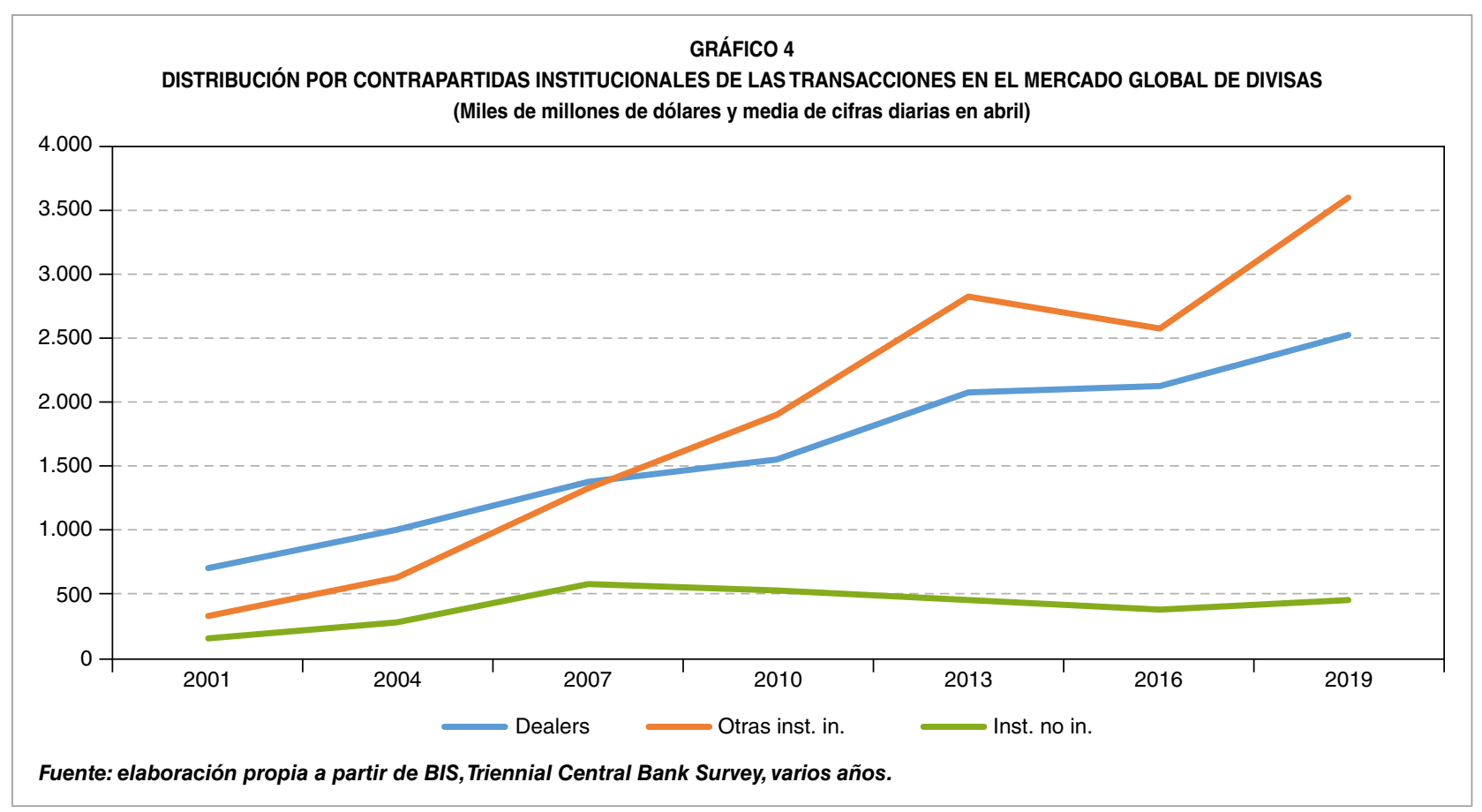


2019), que ha facilitado a todos los intermediarios el acceso directo a los intercambios, dada su mayor flexibilidad, en detrimento de la utilización del mercado interbancario, abierto en general a las entidades de crédito, y no a todos los intermediarios financieros, mercado que sí desempeñó un papel más relevante en la última parte del siglo pasado (Wooldridge, 2019).

Por otra parte, en cuanto al carácter global o transfronterizo de las operaciones realizadas en los mercados mundiales de divisas, podemos comprobar en el Gráfico 5 el predominio de las transfronterizas o cross-border.

Ese predominio las ha llevado a suponer, en algunos de los años contemplados, hasta el $65 \%$ del total de transacciones, como ocurrió en las encuestas de 2010 y 2016, pero que, por lo general, se han movido entre el $55 \%$ y el $60 \%$ del total.

\subsubsection{Concentración del mercado mundial}

En términos generales, podemos comprobar en el Gráfico 6 que es el mercado británico el que logra mayores volúmenes intercambiados de divisas, con más de 3,5 billones de dólares, muy por encima de cualquier otro mercado nacional, pues el más próximo es el norteamericano, que alcanza una cuantía de 1,37 billones. La destacada irrupción de los mercados orientales supone que, tanto Singapur como Hong Kong, seguidos del mercado japonés, se coloquen entre los cinco primeros lugares del mundo, los dos primeros muy igualados entre sí (633 y 632 mil millones de dólares diarios intercambiados, respectivamente).

Además, este mercado de divisas, a nivel de centros financieros, presenta un elevado grado de concentración, pues basta comprobar, en primer lugar, que entre el británico y $\triangleright$

GRÁFICO 5

DISTRIBUCIÓN DE LAS OPERACIONES REALIZADAS EN EL MERCADO GLOBAL DE DIVISAS POR LOCALIZACIÓN DE LAS CONTRAPARTIDAS

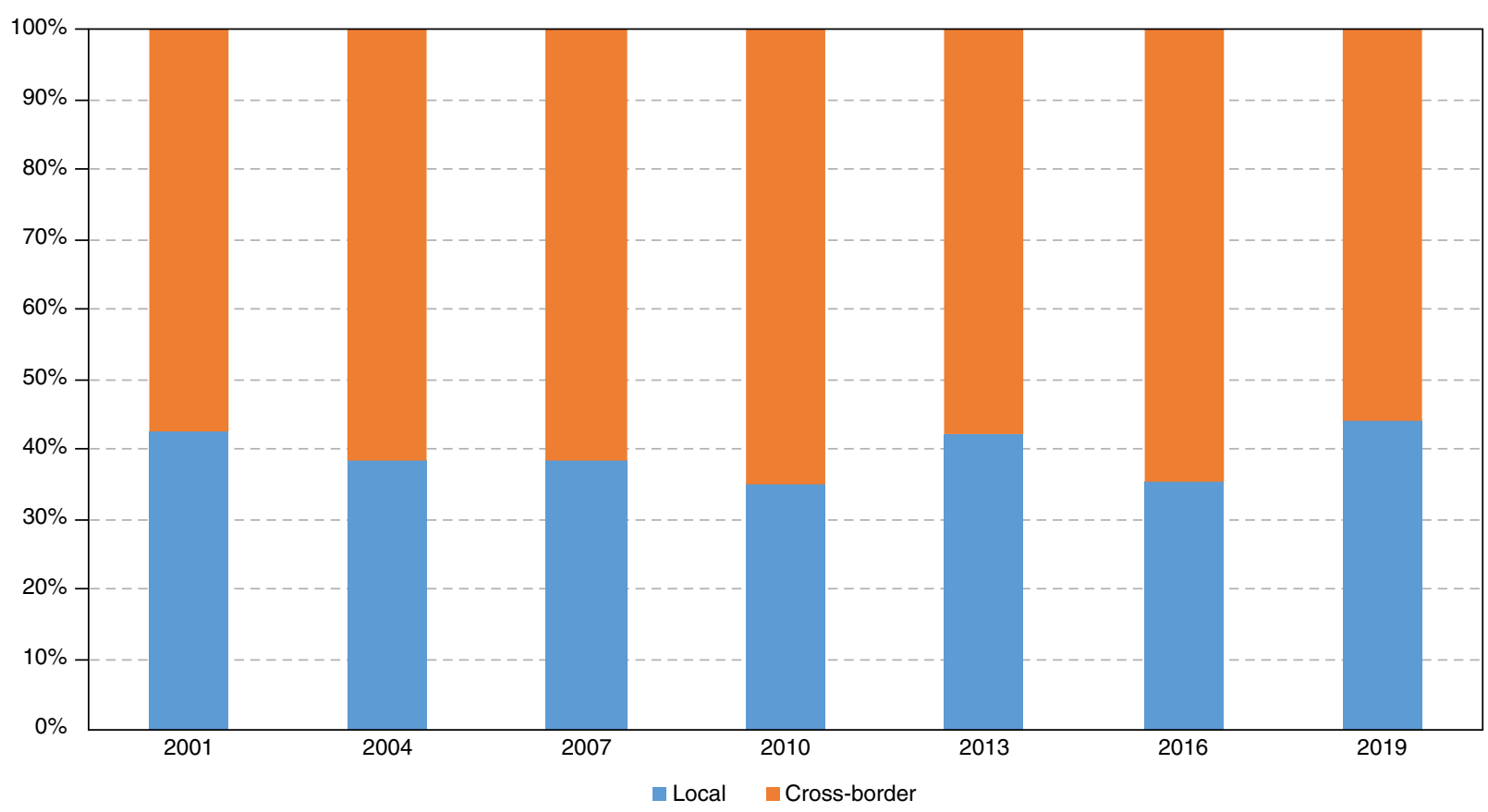

Fuente: elaboración propia a partir de BIS, Triennial Central Bank Survey, varios años. 


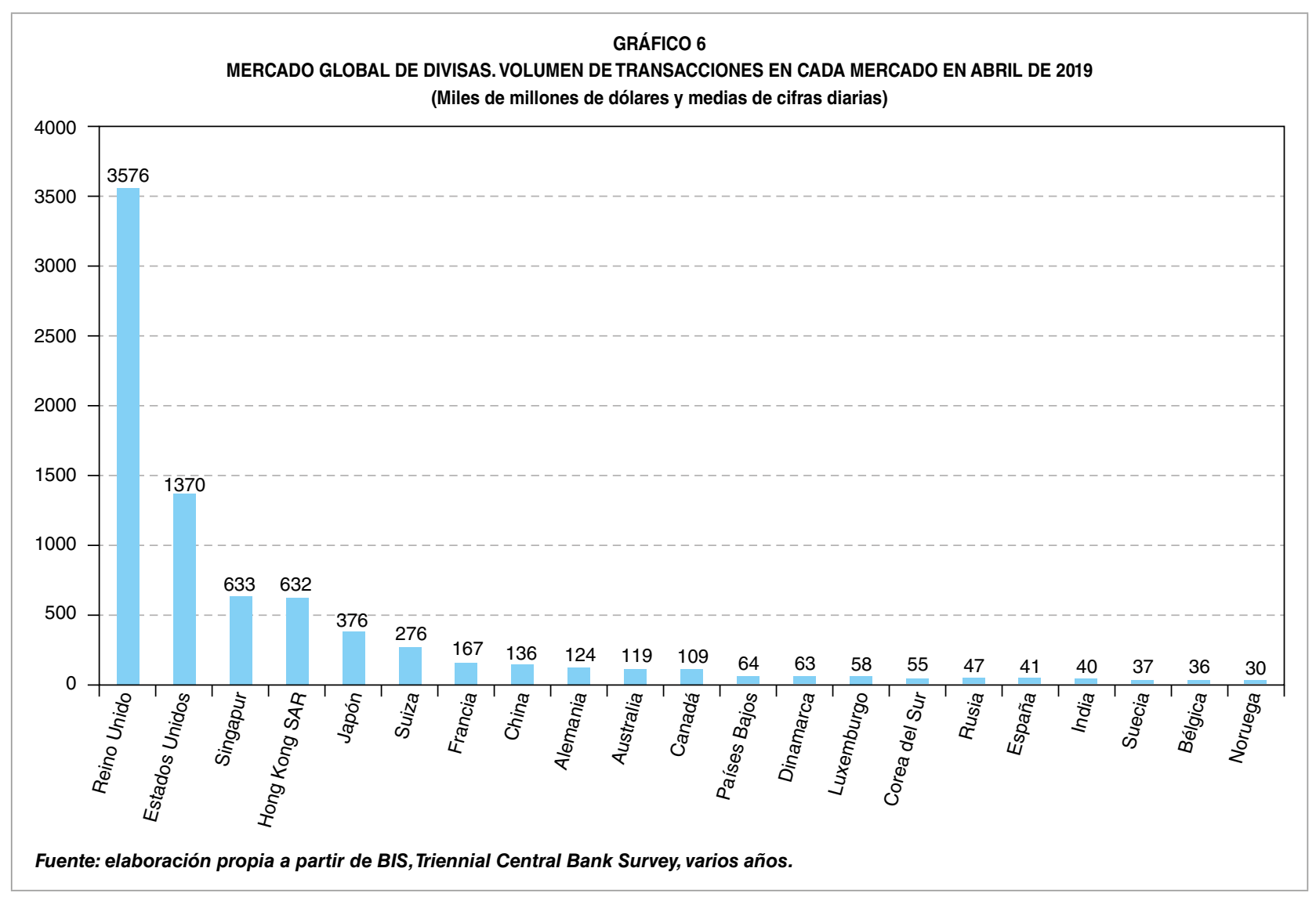

el estadounidense acaparan casi el $60 \%$ del total, y si consideramos además los países asiáticos, ya destacados anteriormente, el nivel de concentración se sitúa en el $80 \%$. Este predominio de los dos primeros mercados se mantiene a lo largo del tiempo, como podemos comprobar en el Gráfico 7, en el que podemos constatar que el nivel de importancia de algunos de los mercados nacionales, a lo largo de lo transcurrido desde finales del siglo pasado, ha cambiado de forma importante, siendo lo más destacado, por un lado, la preponderancia creciente del mercado británico, pues frente a un mínimo de cuota alcanzada en el inicio del siglo $(31,8 \%)$ en la última encuesta concentró, como hemos dicho, más del $43 \%$ de las transacciones mundiales de divisas; por otro, la estabilización, entre el inicio y el final del periodo considerado, del mercado norteamericano, en torno al $16-17 \%$, con limitadas variaciones en las diferentes encuestas realizadas; por otro, la caída de importancia del mercado japonés, tradicionalmente en tercer lugar, en beneficio de otros mercados de su área geográfica, como Singapur y Hong Kong; y, finalmente, la continua pérdida de protagonismo de los países europeos en este mercado.

Por áreas geográficas, en el Cuadro 6 recogemos las cifras correspondientes a las tres últimas encuestas, que nos dan una idea de la ya destacada pérdida de importancia de la región de América del Norte, representada, casi en su totalidad, por el mercado USA; la estabilización de la región occidental de Europa, que, sin embargo, encierra una gran heterogeneidad entre los mercados que la integran, pues el británico ha absorbido una parte significativa de lo que se intermediaba en los $\triangleright$ 


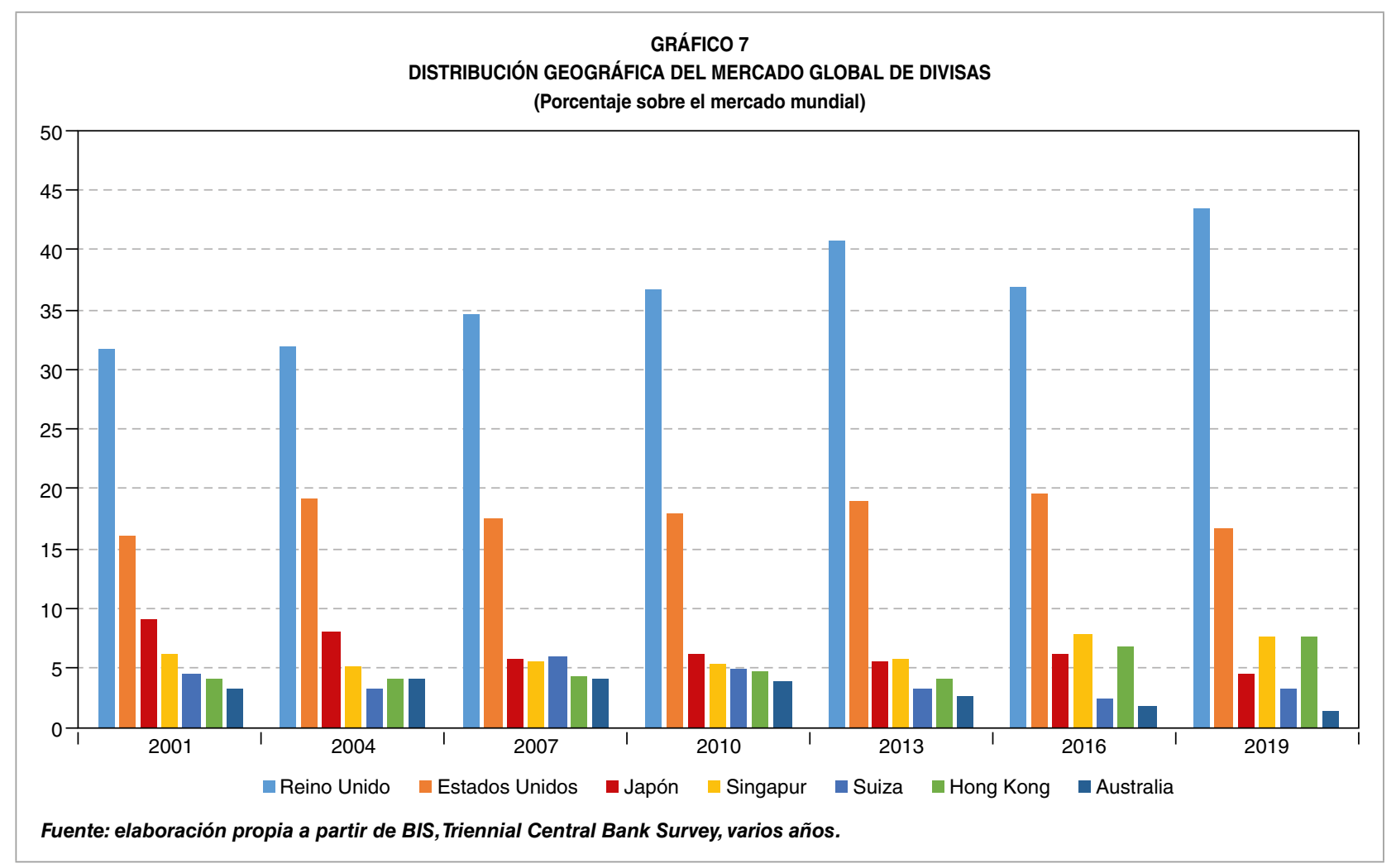

mercados alemán, francés, italiano o español; o el crecimiento de la región Asia-Pacífico, que en tres encuestas ha ganado casi cuatro puntos de la cuota mundial de los intercambios, con la caída, aunque su relevancia es mucho menor, de las regiones latinoamericanas y africana.

\section{Conclusiones}

A lo largo de este trabajo hemos analizado la evolución del mercado mundial de divisas, realizando comparaciones entre encuestas en sus aspectos más relevantes, pero con mayor hincapié en los datos correspondientes a la $\triangleright$

CUADRO 6

DISTRIBUCIÓN REGIONAL DEL MERCADO GLOBAL DE DIVISAS (Miles de millones de dólares y medias diarias en abril)

\begin{tabular}{|c|c|c|c|c|c|c|}
\hline & 2013 & $\%$ s/total & 2016 & $\%$ s/total & 2019 & $\%$ s/total \\
\hline 1. América del Norte & 1.328 & $1.952,9$ & 1.358 & $20,8 \%$ & 1.479 & $17,9 \%$ \\
\hline 2. Europa Occidental & 3.715 & $5.463,2$ & 3.277 & $50,3 \%$ & 4.522 & $54,8 \%$ \\
\hline 3. Asia-Pacífico & 1.434 & $2.108,8$ & 1.714 & $26,3 \%$ & 2.091 & $25,3 \%$ \\
\hline 4. Europa Oriental & 83 & 122,1 & 68 & $1,0 \%$ & 72 & $0,9 \%$ \\
\hline 5. Latinoamérica & 68 & 100,0 & 54 & $0,8 \%$ & 55 & $0,7 \%$ \\
\hline 6. África y Oriente Medio & 43 & 63,2 & 43 & $0,7 \%$ & 35 & $0,4 \%$ \\
\hline Total regiones $(1+2+3+4+5+6)$ & 6.671 & $9.810,3$ & 6.514 & $100,0 \%$ & 8.254 & $100,0 \%$ \\
\hline Ajuste por doble contabilización & -1.314 & - & -1.447 & - & -1.664 & - \\
\hline Volumen global & 5.357 & - & 5.067 & - & 6.590 & - \\
\hline
\end{tabular}


última efectuada a nivel global en 2019 y coordinada por el Banco Internacional de Pagos de Basilea.

En general, podemos señalar, a partir de los datos disponibles, que en la reciente encuesta se han observado cambios importantes y muy rápidos. Así, las transacciones en divisas han pasado a efectuarse con mayor intensidad por medios electrónicos; los participantes se han diversificado, frente al predominio, mucho mayor en otros periodos, de las entidades bancarias de mayor tamaño, lo que ha aumentado la velocidad de los intercambios; y se han desarrollado más posibilidades y facilidades para acceder a una mayor variedad de operaciones.

En este último año el volumen de operaciones ha aumentado un $30 \%$ respecto a las cifras de la encuesta de 2016; con un dólar USA que aparece en más del $88 \%$ de las transacciones, seguido del euro, con el 32,3\%; que, además, son las divisas que más se combinan en el mercado, pues ambas suponen una cuarta parte del conjunto de las mismas; si bien destaca la presencia de monedas de los países emergentes, cuyos intercambios con el dólar ya suponen una de cada cinco operaciones; que, merced a ello, los mercados de esos países, fundamentalmente correspondientes al sudeste asiático, han sido los que más han crecido; que siguen siendo las operaciones swaps las que suponen la mayor parte del mercado, sobre todo en los plazos de hasta una semana; y la contrapartida institucional más representativa corresponde a las otras instituciones financieras de menor tamaño y a las operaciones transnacionales frente a las de carácter doméstico.

Respecto al futuro, los procesos de innovación financiera tan acusados que se siguen viviendo, y que se van a intensificar en próximos años, el desarrollo de las fintech y de las bigtech, la evolución de las criptomonedas, los riesgos sobre la libertad de comercio a nivel mundial, el impacto de determinadas pandemias sobre la evolución económica general, el Brexit o los movimientos políticos desestabilizadores a nivel mundial y en el caso español, que algunos de ellos también han ocurrido, junto a otros, con diferente intensidad en otros periodos de encuestas, convierten en especialmente atractivos los nuevos resultados que puedan obtenerse en las futuras encuestas sobre el mercado mundial y español de divisas.

\section{Bibliografía}

Alcalde-Gutiérrez, E. E., Calvo-Bernardino, A., y Paúl-Gutiérrez, J. (2014). La evolución del mercado global de divisas y su situación en el contexto de la crisis financiera. Boletín Económico de Información Comercial Española (3049), 3-14.

Alcalde-Gutiérrez, E. E., Calvo-Bernardino, A., y Paúl-Gutiérrez, J. (2018). La evolución del mercado global de divisas y su situación tras la crisis financiera. Boletín Económico de Información Comercial Española (3103), 19-32.

Bank for International Settlements (varios años). Triennial Central Bank Survey, diciembre, Basilea.

Bank for International Settlements (2019). International Banking and Financial Market Developments. BIS Quarterly Review, diciembre, Basilea.

Bank for International Settlements (2019). Easing trade tensions lift sentiment. BIS Quarterly Review, diciembre, Basilea.

Calvo-Bernardino, A., y Paúl-Gutiérrez, J. (2008a). El mercado de divisas en España. Cuadernos de Información Económica (206), 103-108. 
Calvo-Bernardino, A., y Paúl-Gutiérrez, J. (2008b). El mercado mundial de divisas: evolución, volumen y composición. Boletín Económico de Información Comercial Española (2938), 19-35.

Casilda Béjar, R. (2020). Los bancos en evolución. Boletín Económico de Información Comercial Española (3119), 43-56.

Granell, F. (2020). El sistema económico internacional en 2019. Boletín Económico de Información Comercial Española (3119), 13-30.

Patel, N., y Xia, D. (2019). Offshore markets drive trading of emerging market currencies. BIS Quarterly Review, 53-68, diciembre, Basilea.
Paúl-Gutiérrez, J., y Calvo-Bernardino, A. (2011). Evolución y situación actual del mercado global de divisas. Boletín Económico de Información Comercial Española (3017), 25-35.

Schrimpf, A., y Sushko, V. (2019 a). Sizing up global foreign exchange markets. BIS Quarterly Review, 21-38, diciembre, Basilea.

Schrimpf, A., y Sushko, V. (2019 b). FX trade execution: complex and highly fragmented. BIS Quarterly Review, 39-52, diciembre, Basilea.

Wooldridge, P. (2019). FX and OTC derivaties markets through the lens fo the Triennial Survey. BIS Quarterly Review, 15-20, diciembre, Basilea. 